\title{
Solución del problema de planeamiento de la expansión de redes de transmisión de energía eléctrica mediante un algoritmo genético y programación lineal y no lineal
}

\author{
Ricardo A. Bolaños*, Carlos A. Correa*\$, Mauricio Granada* \\ * Programa de Ingeniería Eléctrica, Universidad Tecnológica de Pereira, Colombia \\ §e-mail:adrian@ohm.utp.edu.co
}

(Recibido: Septiembre 19 de 2007 - Aceptado: Abril 18 de 2008)

\begin{abstract}
Resumen
En este artículo se propone un enfoque eficiente para la reducción del esfuerzo computacional asociado a la solución del problema de planeamiento de la expansión de redes de transmisión de energía eléctrica. Este enfoque utiliza un método de punto interior no lineal para iniciar la población de un algoritmo genético que resuelve de manera iterativa el problema de inversión. Desde el inicio de la solución, las configuraciones generadas son de alta calidad y se encuentran localizadas en puntos estratégicos del espacio solución de modo que pueden evolucionar hacia regiones óptimas. El plan de inversión del algoritmo genético se evaluó para el sistema de transmisión a través de un método de punto interior lineal. El enfoque propuesto se puso a prueba con los sistemas IEEE de 6 y 24 nodos y con el sistema sur-brasilero de 46 nodos. Se obtuvo un excelente desempeño del algoritmo genético para estos sistemas y se requirió un menor esfuerzo computacional para la solución del problema.
\end{abstract}

Palabras clave: Planeamiento de transmisión, Redespacho, Algoritmos genéticos, Métodos de puntos interiores, Programación lineal, Programación no lineal.

ELECTRICAL ENGINEERING

\section{Solution of the electric power transmission-network expansion planning problem by means of a genetic algorithm and linear and non-linear programming}

\begin{abstract}
In this paper, an efficient approach is proposed for the reduction of the computational effort associated to the solution of the electric power transmission-network expansion planning problem. This approach makes use of a non-linear interior-point method to start the population of a genetic algorithm that iteratively solves the problem of investment. From the beginning of the solution, the generated configurations are of high quality and are located at strategic points of the solution space so that they can evolve towards optimal regions. The investment plan for the genetic algorithm was evaluated on a transmission system through a linear interior-point method. The proposed approach was tested on 6 and 24-bus IEEE systems and on the South Brazilian 46-bus system. An excellent performance of the genetic algorithm was obtained for these systems and a lesser computational effort was required for the solution of the problem.
\end{abstract}

Keywords: Transmission planning, Redispatch, Genetic algorithms, Interior point methods, Linear programming, Non-linear programming. 


\section{Introducción}

El problema de planeamiento de la expansión de redes de transmisión de energía eléctrica tiene como objetivo determinar la cantidad, ubicación y tiempo para la instalación de nuevos equipos del sistema de transmisión con el propósito de satisfacer la demanda futura con un mínimo costo de inversión. El problema puede resolverse mediante un modelo estático como lo muestran Bolaños et al. (2007), Correa et al. (2007) y Gallego (2005), o mediante un modelo dinámico como el planteado por Escobar (2002) y Asada et al. (2005), con uno o varios escenarios de generación y demanda, respectivamente.

Los modelos implementados para la solución del problema en orden de complejidad son: modelos de transportes planteados por Garver (1970) y Gallego et al. (2007), modelos híbridos como los de Escobar (2002) y Gallego et al. (2007), modelo DC desarrollado por Bolaños et al. (2007), Gallego (2005), Sánchez et al. (2005) y Gallego et al. (2007), y el modelo AC desarrollado por Rider et al. (2006).

En este artículo se aborda el problema de la expansión de redes de transmisión mediante el modelo DC, que resulta en un problema de optimización no lineal entero mixto (NLEM) de gran tamaño. Dicho modelo se convierte en un problema de programación lineal (PL) cuando se conoce alguna propuesta de inversión como lo muestran Bolaños et al. (2007), Correa et al. (2007) y Gallego (2005). Bajo este esquema, se resuelven dos problemas, uno de inversión y otro de tipo operativo. Debido al elevado número de alternativas que se deben evaluar durante el proceso iterativo, se plantea previamente la solución del modelo DC mediante el método de puntos interiores (MPI) para programación no lineal (PNL) de modo que el número de líneas por corredor que deben instalarse se consideran variables. La respuesta relajada normalmente se usa para resolver el problema de planeamiento mediante un algoritmo heurístico constructivo (AHC) [Sánchez et al. (2005); Rider (2006)], que usa un índice de sensibilidad para tomar la decisión de adicionar o no un circuito en alguno de los corredores del sistema.
En este artículo, se utiliza un algoritmo genético para resolver el problema de inversión mediante un esquema similar al de Escobar (2002) y cuyas propuestas se evalúan para determinar el punto de operación (flujo de carga) del sistema mediante un problema de PL resuelto a través del método de punto interior como lo hacen Gallego (2005) y Rider (2004). Como una nueva propuesta y mejora, en el presente enfoque se utiliza programación no lineal para resolver el problema relajado. Al obtener la solución (número de circuitos por corredor) en forma de variables continuas, se construye un conjunto de configuraciones que hacen parte de la población inicial del algoritmo genético. De esta forma, la exploración del espacio de soluciones se realiza de forma eficiente desde el inicio del algoritmo, potenciando el desempeño del mismo. Este planteamiento se aleja de los enfoques mencionados anteriormente ya que, en éstos, en ningún caso se combina la solución del modelo DC relajado con la implementación de un algoritmo combinatorial. Por el contrario, los dos se utilizan como herramientas independientes para resolver el problema del planeamiento de la expansión. Finalmente, la metodología implementada se pone a prueba mediante su aplicación a los sistemas IEEE Garver de 6 y 24 nodos y el sistema real sur-brasilero de 46 nodos.

\section{Metodología}

\subsection{Modelo DC para el planeamiento de la transmisión}

El modelo no lineal DC (Sánchez et al., 2005; Rider, 2006), que considera generadores ficticios para evitar no factibilidades, y que es usado para resolver el problema de planeamiento de la expansión de redes de transmisión como un problema relajado es el siguiente:

$$
\text { Minimizar } \sum_{i, j \in \Omega} c_{i j} n_{i j}+\alpha \sum_{i \in N} r_{g_{i}}
$$

sujeto a

$$
\begin{gathered}
S f+g+r_{g}=d \\
f_{i j}-\gamma_{i j}\left(n_{i j}+n_{i j}^{0}\right)\left(\theta_{i}-\theta_{j}\right)=0
\end{gathered}
$$




$$
\begin{gathered}
\left|f_{i j}\right| \leq\left(n_{i j}+n_{i j}^{0}\right) \overline{f_{i j}} \\
\underline{g} \leq g \leq \bar{g} \\
0 \leq n_{i j} \leq \overline{n_{i j}} \\
0 \leq r_{g} \leq d \\
n \text { entero } \\
i j \\
\theta_{i} \text { ilimitado } \\
i, j \in \Omega
\end{gathered}
$$

donde los símbolos $c_{i j}, f_{i j}, \gamma_{i j}, n_{i j}, n_{i j}^{0}, \overline{f_{i j}}$ y $\overline{n_{i j}}$ representan, respectivamente, el costo, el flujo, la susceptancia, el número de circuitos adicionados, el número de circuitos del caso base, el flujo máximo y el número máximo de circuitos por corredor de la rama $i j ; S$ es la matriz de incidencia nodo-elemento, $f$ es un vector cuyos elementos son los flujos $f_{i j}, \theta_{i}$ es el ángulo en el nodo $I$, $\Omega$ es el conjunto de ramas candidatas e $i j$ representa el corredor entre las barras $i$ y $j$; las Ecs. (2) y (3) representan la primera y segunda leyes de Kirchhoff de la red DC equivalente; la Ec.(4) es el conjunto de restricciones de desigualdad asociadas con la capacidad de carga de las líneas y donde $g, g, \bar{g}, d$ y $r_{g}$ son los vectores de generadores y sus valores mínimos y máximos operativos y los vectores ficticios de demandas y generaciones, respectivamente.

Se presenta a continuación el modelo para el caso del problema de PL donde $n_{i j}$ es un dato entregado por un algoritmo genético. Este modelo se usa como un subproblema operativo que minimiza el corte de carga $r_{g}$ sin considerar redespacho:

Minimizar $\alpha \sum_{i \in N} r_{g_{i}}$

sujeto a

$$
\begin{gathered}
S f+r_{g}-r_{c}=D G \\
f_{i j}-\gamma_{i j}\left(n_{i j}+n_{i j}^{0}\right)\left(\theta_{i}-\theta_{j}\right)=0
\end{gathered}
$$

$$
\begin{gathered}
L \leq f_{i j} \leq U \\
0 \leq r_{g} \leq d \\
0 \leq r_{c} \leq g \\
\theta_{i} \text { ilimitado } \\
i j \in \Omega
\end{gathered}
$$

donde $U=-L=\left(n_{i j}+n_{i j}^{0}\right) \overline{f_{i j}} \quad$ y $\quad D G=d-g$. Nótese que para este modelo, $g$ deja de ser variable y se adiciona un vector de cargas ficticias $r_{c}$. Además, las restricciones de desigualdad sólo están asociadas a las variables canalizadas.

Un aspecto importante es que si se desea considerar redespacho dentro del modelo (Gallego, 2005), éste obliga a mantener la generación variable acotada por sus límites operativos. El modelo con redespacho es el siguiente

$$
\text { Minimizar } \quad \alpha_{g} \sum_{i \in N} r_{g_{i}}+\alpha_{c} \sum_{j \in N} r_{c_{j}}
$$

\section{sujeto a}

$$
\begin{gathered}
S f+g+r_{g}-r_{c}=d \\
f_{i j}-\gamma_{i j}\left(n_{i j}+n_{i j}^{0}\right)\left(\theta_{i}-\theta_{j}\right)=0 \\
L \leq f_{i j} \leq U \\
\underline{g} \leq g \leq \bar{g} \\
0 \leq r_{g} \leq d \\
0 \leq r_{c} \leq \bar{g} \\
\theta_{i} \text { ilimitado } \\
i j \in \Omega
\end{gathered}
$$




\subsection{Método de puntos interiores para programación no lineal}

El método de puntos interiores se ajusta bastante bien a la solución de problemas tanto de programación lineal como de programación no lineal, como lo expone ampliamente Rider (2004). Se describe a continuación el método usado para el caso del modelo relajado del planeamiento de la expansión de la transmisión.

\subsubsection{Método primal dual(MPD)}

Una forma canónica de un problema de programación no lineal con restricciones de desigualdad toma la siguiente forma:

$$
\text { Minimizar } f(x)
$$

sujeta a

$$
\begin{gathered}
g(x)=0 \\
h(x) \leq 0 \\
x^{l} \leq I x \leq x^{u}
\end{gathered}
$$

donde $f(x), g(x), h(x), \hat{I} x, x^{u}$ y $x^{l}$ son la función de costos [Ec.(1)], el conjunto de restricciones de igualdad [Ecs. (2-3)] y de desigualdad [Ec.(4)], el conjunto de variables canalizadas [Ec.(5)] y los límites superior e inferior de las variables canalizadas respectivamente. Además, se designan las cantidades $n x, n d x, n d g, n d h$, como el número de variables del problema, número de variables canalizadas, número de restricciones de igualdad y el número de restricciones de desigualdad, respectivamente.

Usando las variables de holgura $\left(s_{i}>0\right)$ para transformar las restricciones de desigualdad en restricciones de igualdad e introduciendo las condiciones de no negatividad en la función objetivo como términos de barrera logarítmica y finalmente llevando las restricciones de igualdad a la función objetivo, se obtiene la función lagrangiana $L_{u}$ así:

$$
\begin{aligned}
L_{u}= & f(x)-\mu^{k} \sum_{j=1}^{n d h}\left(\ln s_{2 j}\right)-\mu^{k} \sum_{j=1}^{n d x}\left(\ln s_{3 j}+\ln s_{4 j}\right) \\
& -y^{T} g(x)-z_{2}^{T}\left[-h(x)-s_{2}\right]-z_{3}^{T}\left(-s_{3}-s_{4}-x^{l}+x^{u}\right) \\
& -z_{4}^{T}\left(-\hat{I} x-s_{4}+x^{u}\right)
\end{aligned}
$$

donde $\mu^{k}$ es un parámetro de barrera que decrece en forma monótona a cero en el proceso iterativo. $\mathrm{Si}$ se aplican las condiciones necesarias de optimalidad de primer orden de Karush-KuhnTucker (KKT), $\nabla L_{\mu}=0$, se obtiene un conjunto de ecuaciones denominado $F(w)=0$. Si este conjunto se resuelve mediante el método de Newton, se obtiene:

$$
\left[J_{F}\left(w^{k}\right)\right] \Delta w^{k}=-F\left(w^{k}\right)
$$

Los elementos de la matriz $J_{F}\left(w^{k}\right)$ se obtienen con las derivadas parciales de segundo orden de $F(w)$. Definiendo a $S_{i}$ y $Z_{i}$ como matrices diagonales con entradas $s_{i}$ y $z_{i}$, respectivamente, la Ec.(19) puede escribirse en forma extendida así:

$$
\left[\begin{array}{cccccccc}
Z_{2} & 0 & 0 & 0 & 0 & S_{2} & 0 & 0 \\
0 & Z_{3} & 0 & 0 & 0 & 0 & 0 & 0 \\
0 & 0 & Z_{3}+Z_{4} & S_{4} & S_{4} & 0 & 0 & 0 \\
0 & I & I & 0 & 0 & 0 & 0 & 0 \\
0 & 0 & I & 0 & 0 & 0 & \hat{I} & 0 \\
I & 0 & 0 & 0 & 0 & 0 & J_{h} & 0 \\
0 & 0 & 0 & \hat{I}^{T} & 0 & J_{h}^{T} & \nabla_{x}^{2} L_{u} & -J_{g}^{T} \\
0 & 0 & 0 & 0 & 0 & 0 & -J_{g} & 0
\end{array}\right]\left[\begin{array}{c}
\Delta s_{2} \\
\Delta s_{3} \\
\Delta s_{4} \\
\Delta z_{3} \\
\Delta z_{4} \\
\Delta z_{2} \\
\Delta x \\
\Delta y
\end{array}\right]=\left[\begin{array}{c}
\mu^{k} e-S_{2} z_{2} \\
\mu^{k} e-S_{3} z_{3} \\
\mu^{k} e-S_{4}\left(z_{3}+z_{4}\right) \\
-S_{3}-s_{4}-x^{l}+x^{u} \\
-\hat{I} x-s_{4}+x^{u} \\
-h(x)-s_{2} \\
-\nabla f+J_{g}^{T} y-J_{h}^{T} z_{2}-\hat{I}^{T} z_{4} \\
g(x)
\end{array}\right]
$$


donde $\nabla f \in R^{n x} \quad$ es el gradiente de la función objetivo y $J_{g} \in R^{n d g}$ y $J_{h} \in R^{n d h}$ son las matrices jacobianas de las restricciones de igualdad y desigualdad, respectivamente, $y$

$\nabla_{x}^{2} L_{\mu}=H_{f}\left(x^{k}\right)-\sum_{j=1}^{n d g} y_{j}^{k} H_{g j}\left(x^{k}\right)+\sum_{j=1}^{n d h} z_{2 j}^{k} H_{h j}\left(x^{k}\right)$

es el término que complica el problema de programación no lineal, dado que exige el cálculo de $n d g+n d h+1$ matrices hessianas en cada iteración.

El punto inicial debe cumplir con la condición $s_{2}^{0}, z_{2}^{0}, s_{3}^{0}, s_{4}^{0}, z_{3}^{0},\left(z_{3}^{0}+z_{4}^{0}\right)>0$. Para ello, dado que el proceso de convergencia es sensible al punto inicial, una manera de asignar valores iniciales a las variables primales consiste en tomar el punto medio entre los límites superior e inferior de aquellas variables canalizadas y ceros para las variables libres. Las variables $y_{j}$ son 0 o -1 al inicio del proceso, y para las variables de holgura primales se tiene que:

$$
\begin{aligned}
& s_{2 i}^{0}=\left\|h\left(x^{0}\right)\right\| \\
& s_{3 j}^{0}=\min \left\{\max \left\{\tau x_{j}^{\Delta}, \hat{I} x^{0}-x_{j}^{l}\right\},(1-\tau) x_{j}^{\Delta}\right\} \\
& s_{4 j}^{0}=x_{j}^{\Delta}-s_{3 j}^{0}
\end{aligned}
$$

Típicamente, $\tau=0.25$; además, $x^{\Delta}=x^{u}-x^{l}$ $\mathrm{y}$ a las variables de holgura duales se les asigna valores iniciales del siguiente modo:

$$
\begin{aligned}
& z_{2}^{0}=\mu^{0}\left(S_{2}^{0}\right)^{-1} e \\
& z_{3}^{0}=\mu^{0}\left(S_{3}^{0}\right)^{-1} e \\
& z_{4}^{0}=\mu^{0}\left(S_{4}^{0}\right)^{-1} e-z_{3}^{0}
\end{aligned}
$$

Después de obtener las direcciones $\Delta w^{k}$ de la solución de la Ec.(20), los nuevos valores de las variables primales, duales y de holgura para la iteración $k+1$ se obtienen por medio de las siguientes expresiones:

$$
\begin{aligned}
& x^{k+1}=x^{k}+\gamma \alpha_{p}^{k} \Delta x^{k} \\
& y^{k+1}=y^{k}+\gamma \alpha_{d}^{k} \Delta y^{k}
\end{aligned}
$$

$$
\begin{aligned}
& s_{i}^{k+1}=s_{i}^{k}+\gamma \alpha_{p}^{k} \Delta s_{i}^{k} \\
& z_{i}^{k+1}=z_{i}^{k}+\gamma \alpha_{d}^{k} \Delta z_{i}^{k} \\
& \text { para } i=2,3,4
\end{aligned}
$$

donde $\gamma \in(0,1)$ y es un parámetro de seguridad para garantizar que el próximo punto satisfaga las condiciones de no negatividad. Un valor típico tanto para PL como para PNL es $\gamma=0.99995$. Los escalares $\alpha_{p}^{k} \mathrm{y} \alpha_{d}^{k}(0,1]$, son las longitudes de paso primal y dual, respectivamente, para la iteración $k$. En programación no lineal, se acostumbra elegir $\alpha=\min \left\{\alpha_{p}^{k}, \alpha_{d}^{k}\right\}$, donde $\alpha_{p}^{k}, \alpha_{d}^{k}$ se obtienen del siguiente modo:

$$
\alpha_{p}^{k}=\min _{i j}\left\{1, \min _{\Delta s_{2 i}^{k}<0}\left(\frac{-s_{2 i}^{k}}{\Delta s_{2 i}^{k}}\right), \min _{\Delta s_{3 j}^{k}<0}\left(\frac{-s_{3 j}^{k}}{\Delta s_{3 j}^{k}}\right), \min _{\Delta s_{j j}^{k}<0}\left(\frac{-s_{4 j}^{k}}{\Delta s_{4 j}^{k}}\right)\right\}
$$

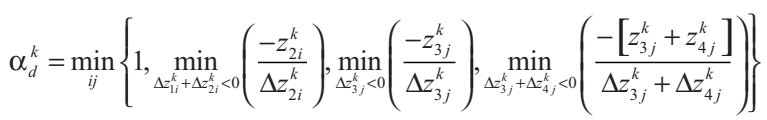

E1 valor residual de la condición de complementariedad tiende monótonamente a cero durante el proceso iterativo. La brecha de complementariedad $\rho^{k}$ junto con el parámetro de barrera $\mu^{k}$ se calculan en cada iteración $k$ así: 


$$
\begin{gathered}
\rho^{k}=\left(z_{2}^{k}\right)^{T} s_{2}^{k}+\left(z_{3}^{k}\right)^{T} s_{3}^{k}+\left(z_{3}^{k}+z_{4}^{k}\right)^{T} s_{4}^{k} \\
\mu^{k+1}=\beta^{k} \frac{\rho^{k}}{2(n d x+n d h)}
\end{gathered}
$$

donde $\beta \in(0,1)$ y es un parámetro de centralización. Para compensar los objetivos de reducir $\mu^{k}$ y mejorar la dirección central, $\beta^{k}$ se escoge como $\beta^{k+1}=\max \left\{0.95 \beta^{\mathrm{k}}, 0.1\right\}$, con $\beta^{0}=0.2$. Generalmente, $\mu^{0}=0.1,1$ o 10 .

El sistema de ecuaciones representado por la Ec.(20) debe ser resuelto hasta que cada uno de los siguientes criterios de convergencia se cumplan:

Factibilidad primal,

$$
\begin{aligned}
& \max \left\{\|g(x)\|_{\infty}, \max \left\{x^{l}-x^{k}\right\}, \max \left\{x^{k}-x^{u}\right\},\right. \\
& \left.\max \left\{h^{l}-h\left(x^{k}\right)\right\}, \max \left\{h\left(x^{k}\right)-h^{u}\right\}\right\} \leq \epsilon_{f}
\end{aligned}
$$

Factibilidaddual,

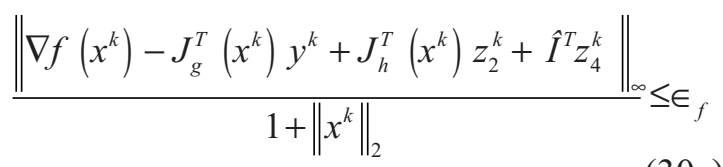

Condición de optimalidad,

$$
\frac{\rho^{k}}{1+\left\|x^{k}\right\|_{2}} \leq \epsilon_{0}
$$

Desvio de la función objetivo,

$$
\frac{\left|f\left(x^{k}\right)-f\left(x^{k-1}\right)\right|}{1+\left|f\left(x^{k}\right)\right|} \leq \epsilon_{f}
$$

\subsection{Método de puntos interiores para programación lineal}

A continuación se presenta el método primal dual para programación lineal.

\subsubsection{Método primal dual (MPD)}

El problema del planeamiento de la expansión de redes de transmisión tal como está descrito por las Ecs.(6-9) y (10-12) puede expresarse de la siguiente forma canónica:

Minimizar $\quad c^{T} x$

sujeto a

$$
A x=b
$$

$$
x^{l} \leq \hat{I} x \leq x^{u}
$$

donde $c^{T} x, A x=b, \hat{I} x, x^{u}, x^{l}$ son, respectivamente, las funciones objetivo definidas por las Ecs. (6) y (10), el conjunto de restricciones de igualdad dadas por las Ecs. (7) y (8) y (11) y (12), el conjunto de variables canalizadas mostradas en las Ecs. (9) y (13), y los límites superior e inferior de las variables canalizadas.

La función lagrangiana, $L_{\mu}$, para este problema está dada por:

$$
\begin{gathered}
L_{\mu}=c^{T} x-\mu^{k} \sum_{j=1}^{n d x}\left(\ln s_{3 j}+\ln s_{4 j}\right)-y^{T}(A x-b) \\
-z_{3}^{T}\left(-s_{3}-s_{4}-x^{l}+x^{u}\right)-z_{4}^{T}\left(-\hat{I} x-s_{4}+x^{u}\right)
\end{gathered}
$$

Siguiendo el mismo procedimiento descrito anteriormente, se obtiene el siguiente sistema de ecuaciones para el cálculo de las direcciones de búsqueda $\Delta w^{k}$ por el método de Newton:

$$
\left[\begin{array}{cccccc}
Z_{3} & 0 & S_{3} & 0 & 0 & 0 \\
0 & Z_{3}+Z_{4} & S_{4} & S_{4} & 0 & 0 \\
I & I & 0 & & 0 & 0 \\
0 & I & 0 & 0 & I & 0 \\
0 & 0 & 0 & I^{T} & 0 & -A^{T} \\
0 & 0 & 0 & 0 & -A & 0
\end{array}\right]\left[\begin{array}{l}
\Delta s_{3} \\
s_{4} \\
\Delta z_{3} \\
\Delta z_{4} \\
\Delta x \\
\Delta y
\end{array}\right]=\left[\begin{array}{l}
\mu^{k} e-S_{3} z_{3} \\
\mu^{k} e-S_{4}\left(z_{3}+z_{4}\right) \\
-S_{3}-S_{4}-x^{l}+x^{u} \\
-\hat{I} x-S_{4}+x^{u} \\
-c+A^{T} y-I^{T} z_{4} \\
A x-b
\end{array}\right]
$$


Como el punto inicial debe cumplir con la condición $s_{3}^{0}, s_{4}^{0}, z_{3}^{0},\left(z_{3}^{0}+z_{4}^{0}\right)>0$, a las variables del problema, al igual que a las variables de holgura primales y duales se les asigna valores iniciales de la misma forma reportada por Bolaños et al. (2007). Después de obtener las direcciones $\Delta w^{k}$ de la Ec. (35), los nuevos valores de las variables para la iteración $k+1$ se actualizan mediante la siguiente fórmula de recurrencia:

$$
w^{k+1}=w^{k}+\gamma \alpha_{i}^{k} \Delta w^{k}
$$

El escalar $\alpha_{i}^{k}$ toma valores $\alpha_{p}^{k}$ y $\alpha_{d}^{k} \in(0,1]$, para las longitudes del paso primal y dual, respectivamente, que se obtienen con el mismo enfoque de Bolaños et al (2007).

En este caso, la brecha de complementariedad $\rho^{k} \mathrm{y}$ el parámetro de barrera $\mu^{k+1}$ se calculan en cada iteración $k$ mediante las siguientes expresiones:

$$
\begin{gathered}
\rho^{k}=\left(z_{3}^{k}\right)^{T} s_{3}^{k}+\left(z_{3}^{k}+z_{4}^{k}\right)^{T} s_{4}^{k} \\
\mu^{k+1}=\beta^{k} \frac{\rho^{k}}{2 n d x}
\end{gathered}
$$

El sistema de ecuaciones dado por la Ec. (35) debe resolverse hasta que los mismos criterios de convergencia descritos ampliamente por Bolaños et al. (2007) se cumplan.

\subsection{Incorporación del MPI al planeamiento de la expansión}

Cuando se resuelve el modelo DC relajado mediante el MPI para PNL, el resultado de interés es el número de líneas por corredor $n_{i j}$ requerido para satisfacer la demanda. Pero en este punto existe una problemática adicional, que consiste en que $n_{i j} \in \mathbb{R}$, lo cual realmente corresponde a tener una solución no factible ya que, como se explicó antes, el número de líneas por corredor debe necesariamente ser entero. A pesar de esta no factibilidad, la solución del problema de PNL contiene información de gran importancia sobre los corredores que con alta probabilidad pueden estar en la solución final, hecho que es aprovechado para generar configuraciones factibles mediante diferentes combinaciones a partir de los valores reales de $n_{i j}$.

Cuando el algoritmo genético empieza su evolución con el objetivo de minimizar el costo de inversión, las diferentes configuraciones presentes en la población deben ser analizadas mediante un flujo de carga que determine si la propuesta de inversión evaluada puede satisfacer la demanda. Esta condición operativa del sistema se encuentra mediante la implementación del MPI lineal sobre el modelo DC para planeamiento. De esta forma, se puede determinar si la propuesta presenta o no corte de carga y dicho valor se puede emplear para penalizar la función objetivo que utiliza el algoritmo genético.

\subsection{Algoritmos genéticos}

La idea fundamental de los algoritmos genéticos (AG) consiste en simular el comportamiento genético de los seres sexuados y ellos permiten resolver problemas combinatoriales de gran tamaño como el aquí tratado. La idea central del AG incorporado a la solución del problema de planeamiento la amplía Escobar (2002), y consiste en entregarle al problema operativo propuestas de adición de circuitos para que sea evaluado su corte de carga, y son atractivas aquellas para las que dicho corte es nulo dado que no penaliza la función objetivo dada por las Ecs. (6) y (10). La Figura 1 ilustra la forma en que operan los problemas de inversión y operación en la solución del problema de planeamiento.

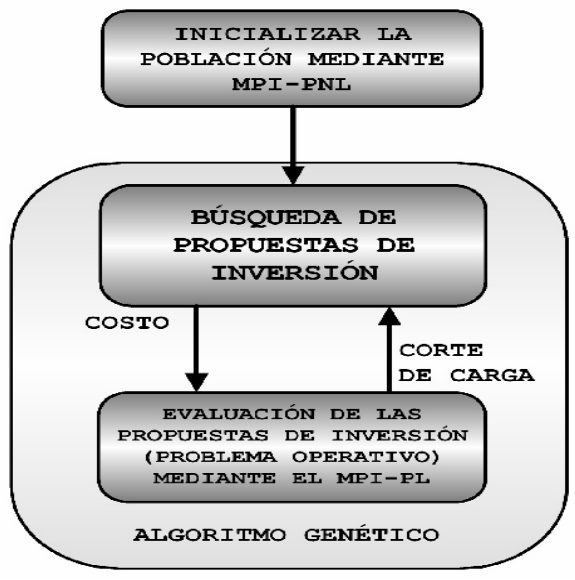

Figura 1. Esquema del algoritmo propuesto. 
Nótese que el problema de inversión (AG) entrega una configuración entera $n_{i j}$ al problema operativo (MPI-PL) para que éste evalúe su corte de carga $r_{g}$ y decida sobre la factibilidad de dicha propuesta. El algoritmo genético que se implementó para los sistemas de bajo tamaño (IEEE-24 bus y Garver) es el denominado básico y usa los operadores de selección por torneo, recombinación y mutación propios de esta metodología expuestos ampliamente por Escobar (2002), y de otra parte para el sistema sur-brasilero de 46 barras se implementó el AG especializado de Chu-Beasley debido al incremento en la dificultad de este sistema de prueba que presenta la característica de ser real y altamente aislado en su configuración base. El AG de Chu-Beasley mantiene la diversidad entre los individuos de la población y admite sólo un individuo para incluir en la población por ciclo generacional, disminuyendo de esta forma en gran medida el esfuerzo computacional al evaluar menor número de problemas PL. Para el caso de la selección por torneo, se intentan mantener dentro de la población aquellas configuraciones factibles o de menor corte de carga, permitiendo pequeños grados de infactibilidad $r_{g} \neq 0$. La recombinación se realiza entre aquellos padres que obtienen derechos para generar los descendientes del próximo ciclo generacional. La mutación se realiza considerando una probabilidad dada por $\rho_{m}$ (denominada tasa de mutación), y se decide sobre la adición o eliminación de circuitos en algunos corredores de las diferentes configuraciones de la población, seleccionados de manera aleatoria tratando de mantener un adecuado nivel de diversidad. El esquema completo del algoritmo implementado para la solución del problema del planeamiento de la transmisión se muestra en la Figura 1.

\section{Resultados y discusión}

Los sistemas de prueba de pequeño tamaño considerados son IEEE de 6 (Garver, 1970) y 24 nodos. La metodología propuesta se implementa en MatLab ${ }^{\circledR}$ 7.0. Como se mencionó anteriormente, la solución relajada del problema PNL es aprovechada en este trabajo para generar un conjunto de individuos que hace parte de la población inicial del algoritmo genético. Dado que la solución del problema relajado es continua, ésta se lleva a sus valores enteros superiores (debido a que la solución relajada es necesariamente menor o igual a la solución entera) con el fin de obtener una configuración (individuo) de buena calidad y posteriormente se crean nuevos individuos mutando algunas de las posiciones en las que existen o no circuitos. Para llevar a cabo la anterior mutación, se elige de forma aleatoria un corredor $i j$, y también aleatoriamente se adiciona una línea, si $n_{i j}+n_{i j}^{0}<n_{i j \max }$ o se elimina si $n_{i j}+n_{i j}^{0} \geq 1$. De esta forma, se crea una población inicial diversa en torno a un punto que se espera sea el óptimo global del problema.

\subsection{Soluciones encontradas sin considerar redespacho}

En la Tabla 1 se presentan los resultados arrojados por el MIP no lineal para las propuestas de adición de circuitos sobre el caso base, sin redespacho y para cada uno de los sistemas de prueba.

Una vez generada la población inicial, el algoritmo genético llega a la solución óptima de cada problema en muy pocos ciclos generacionales, y el tiempo de cómputo es igualmente pequeño. Para el sistema de Garver la solución arrojada por el PNL es tan cercana a la

Tabla 1. Resultados obtenidos mediante el modelo relajado sin redespacho.

\begin{tabular}{cc}
\hline $\begin{array}{c}\text { Sistema Garver } \\
\text { costo }=180,313 \text { USD }\end{array}$ & $\begin{array}{c}\text { Sistema IEEE 24 Nodos } \\
\text { costo }=87,428 \text { USD }\end{array}$ \\
\hline$n_{2-6}=3.5062 ; n_{3-5}=0.8406 ; n_{4-6}=1.9438$ & $n_{01-05}=0.1413 ; n_{03-24}=0.0622 ; n_{06-10}=0.6034$ \\
& $n_{07-08}=2.0000 ; n_{10-12}=0.3025 ; n_{14-16}=0.4524$ \\
\hline
\end{tabular}


solución entera que basta con la asignación de valores iniciales propuesta para que la configuración óptima esté presente en esta población. Para el sistema IEEE de 24 nodos se ejecutó el algoritmo en 5 ocasiones, para una tasa de mutación de $2 \%$ y diferente número de individuos. La anterior información y los respectivos tiempos de cómputo se condensan en la Tabla 2.

La Tabla 3 presenta la solución óptima obtenida para cada sistema de prueba y la configuración correspondiente.

Es interesante comparar los datos de las Tablas 1 y 3 dado que las configuraciones propuestas por el modelo relajado contienen en un elevado porcentaje los corredores que hacen parte de la solución óptima, por lo que el esfuerzo del algoritmo genético se ve reducido en gran medida.

\subsection{Soluciones encontradas considerando redespacho}

Cuando se considera redespacho (generación variable), es posible resolver el problema de planeamiento partiendo de la configuración base o considerando el sistema aislado, sin elementos en su topología inicial. Los resultados arrojados por el MPI para el problema PNL, ejecutado sobre el sistema de Garver se presentan en la Tabla 4.

Para el sistema de Garver considerando topología base, nuevamente la solución arrojada por el problema PNL es tan cercana a la solución entera que basta con la asignación inicial de valores propuesta para que la configuración óptima esté presente en esta población. Para el caso en que no se considera topología base (sistema aislado), el algoritmo genético encuentra la solución en pocas iteraciones. Los resultados obtenidos para las soluciones óptimas se presentan en la Tabla 5.

Tabla 2 Resultados obtenidos para el sistema de 24 nodos.

\begin{tabular}{|c|c|c|c|c|c|c|}
\hline \multirow{2}{*}{10 individuos } & \# Ciclos & 2 & 9 & 2 & 3 & 3 \\
\cline { 2 - 7 } & $\mathrm{t}(\mathrm{s})$ & 3.59 & 15.75 & 3.50 & 5.17 & 5.28 \\
\hline \multirow{2}{*}{20 individuos } & \# Ciclos & 3 & 2 & 2 & 2 & 4 \\
\cline { 2 - 7 } & $\mathrm{t}(\mathrm{s})$ & 10.86 & 7.42 & 6.94 & 7.12 & 14.09 \\
\hline \multirow{2}{*}{30 individuos } & \# Ciclos & 2 & 3 & 2 & 2 & 6 \\
\cline { 2 - 7 } & $\mathrm{t}(\mathrm{s})$ & 10.59 & 16.01 & 10.50 & 10.75 & 32.57 \\
\hline
\end{tabular}

Tabla 3. Solución óptima de los sistemas de prueba sin redespacho.

\begin{tabular}{|c|c|}
\hline $\begin{array}{c}\text { Sistema Garver } \\
\text { costo }=200,000 \text { USD }\end{array}$ & $\begin{array}{l}\text { Sistema IEEE 24 Nodos } \\
\text { costo }=152,000,000 \text { USD }\end{array}$ \\
\hline$n_{2-6}=4 ; n_{3-5}=1 ; n_{4-6}=2$ & $n_{06-10}=1 ; n_{07-08}=2 ; n_{10-12}=1 ; n_{14-16}=1$ \\
\hline
\end{tabular}

Tabla 4. Resultados obtenidos mediante el modelo relajado con redespacho.

\begin{tabular}{|c|l|}
\hline $\begin{array}{c}\text { Sistema Garver (considerando topología base) } \\
\text { costo }=99,000 \text { USD }\end{array}$ & $\begin{array}{c}\text { Sistema Garver (sin considerar topología base) } \\
\text { costo }=153,000 \text { USD }\end{array}$ \\
\hline$n_{2-3}=0.1818 ; n_{2-6}=1.0273$ & $n_{1-5}=0.700 ; n_{2-3}=1.500 ; n_{2-6}=0.900$ \\
$n_{35}=1.0182 ; n_{46}=1.4727$ & $n_{35}=1.700 ; n_{46}=1.600$ \\
\hline
\end{tabular}


Tabla 5. Solución óptima de los sistemas de prueba con redespacho.

\begin{tabular}{|c|c|}
\hline $\begin{array}{c}\text { Sistema Garver (considerando topología base) } \\
\text { costo }=110,000 \text { USD }\end{array}$ & $\begin{array}{c}\text { Sistema Garver (sin considerar topología base) } \\
\text { costo }=190,000 \text { USD }\end{array}$ \\
\hline$n_{3-5}=1 ; n_{4-6}=3$ & $n_{1-5}=1 ; n_{2-3}=2 ; n_{2-6}=1 ; n_{3-5}=2 ; n_{4-6}=2$ \\
\hline
\end{tabular}

Para el sistema sur-brasilero de 46 nodos se procede de manera similar: se genera una población a partir de la solución relajada, y en este caso para un conjunto de soluciones de dicha población, se bloquean aleatoriamente $\left(0<n_{i j}<0\right)$ algunas casillas haciendo que la población inicial gane en diversidad conservando la característica determinística que le otorga el problema de PNL. Para este sistema se utilizó una población de 40 individuos y la mejor solución obtenida fue de 72,870,000 USD después de 2466 PLs, con la siguiente configuración:

$$
\begin{aligned}
& n_{02-05}=1, \quad n_{05-06}=2, \quad n_{13-20}=1, \quad n_{20-21} \\
& =2, \quad n_{20-23}=1, \quad n_{42-43}=1, \quad n_{46-06}=1
\end{aligned}
$$

\section{Conclusiones y recomendaciones}

El problema del planeamiento de la expansión de la transmisión es un problema de gran tamaño, de difícil solución y que hace parte del grupo de los problemas NP-completos, presentando una alta explosión combinatorial debido al número de posibles soluciones que deben ser evaluadas para encontrar el óptimo. Bajo este esquema, se debe emplear una técnica que pueda realizar una búsqueda inteligente como en los denominados algoritmos combinatoriales. Dichas técnicas tienen un mejor desempeño si se combinan con el conocimiento previo que se tenga del problema y si se incorporan otras técnicas que complementen las características de dichos algoritmos.

La propuesta presentada potencia la utilización de las técnicas combinatoriales basadas en población ya que gran parte del esfuerzo que deben realizar los algoritmos para generar poblaciones de alta calidad disminuye debido al esquema de asignación de valores iniciales mediante la técnica exacta de programación no lineal.
Los tiempos de cómputo y ciclos generacionales requeridos para encontrar las respuestas óptimas son pequeños a pesar de emplear un algoritmo genético básico. Este hecho abre el camino para explorar otras técnicas que puedan aprovechar una asignación inteligente de valores iniciales con la respuesta arrojada por el problema de PNL.

La característica del algoritmo genético de realizar cruzamiento de diferentes alternativas de solución permite a la soluciones hijas heredar características que pueden ser de alta calidad y hacer que la población sea mejor en cada ciclo. Además, dichas soluciones hijas pueden ser mejoradas o empeoradas por medio de la mutación; de hecho, para el caso planteado se debe tener cuidado con la tasa de mutación escogida ya que una tasa de mutación alta puede hacer que la población empeore en cada ciclo. Lo anterior se debe a que la población inicial es de muy alta calidad y es conveniente realizar cambios suaves a los individuos.

\section{Referencias bibliográficas}

Asada, E. N., Carreño, E., Romero, R., \& García, A. V. (2005). A branch-and-bound algorithm for the multi-stage transmission expansion planning. In Proceedings of the 2005 General Meeting of the IEEE Power Engineering Society, Vol. 1, p. 171-176.

Bolaños, R. A., Correa, C. A., \& Garcés-Ruiz, A. (2007). Planeamiento de la expansión de la transmisión considerando contingencias mediante el algoritmo multiobjetivo NSGA-II. Revista Scientia et Tecnica, Año XIII (37), 13-18.

Correa, C.A., Bolaños, R.A., \& Garcés-Ruiz, A. (2007). Modelo multiobjetivo para el planeamiento de la transmisión usando una técnica evolutiva y puntos interiores. Revista Scientia et Tecnica, Año XIII (35), 43-48. 
Escobar, A. (2002). Planeamiento dinámico de la transmisión de sistemas de transmisión usando algoritmos combinatoriales. Tesis de Maestría, Facultad de Ingeniería Eléctrica, Universidad Tecnológica de Pereira, Pereira, Colombia.

Gallego R. A., Monticelli A., \& Romero R. (1998). Comparative studies on nonconvex optimization methods for transmission network expansion planning. IEEE Transactions on Power Systems $13(3), 822-828$.

Gallego, L. A. (2005). Planeamiento de la expansión de redes de transmisión de energía eléctrica considerando contingencias. Tesis de Maestría, Facultad de Ingeniería Eléctrica, Universidad Tecnológica de Pereira, Pereira, Colombia.

Gallego, R. A., Escobar, A., \& Romero, R. A. (2006). Técnicas de optimización combinatorial. Primera Edición, Universidad Tecnológica de Pereira, Pereira, Colombia.

Gallego, R. A., Escobar, A., Romero, R., \& Monticelli, A. (2007). Planeamiento de la expansión de sistemas de transmisión de energía eléctrica. Universidad Tecnológica de Pereira, Pereira, Colombia.
Garver L. L. (1970). Transmission network estimation using linear programming. IEEE Transactions on Power Apparatus and Systems 89 (7), 1688-1697.

Rider, M. J. (2004). Método de puntos interiores para optimización en sistemas eléctricos. Universidad Tecnológica de Pereira, Pereira, Colombia.

Rider, M. J (2006). Planejamento da expansão de sistemas de transmissão usando os modelos CC-CA e tecnicas de programação não-linear. Tese de Doutorado, Engenharia Eletrica e de Computação, Universidade Estadual de Campinas, Brasil.

Rider, M. J., Gallego, L. A., Romero, R., \& Garcia, A. V. (2006). Heuristic algorithm to solve the short term transmission network expansion planning. In Proceedings of the 2007 General Meeting of the IEEE Power Engineering Society, p. 1-7.

Sanchez, I.G., Romero, R., Mantovani, J.R.S., \& Rider, M. J. (2005). Transmission-expansion planning using the DC model and nonlinearprogramming technique. IEE Proceedings on Generation, Transmission and Distribution 152 (6), 763-769. 